\title{
A utilização da técnica de lente térmica para a análise das propriedades térmicas de materiais transparentes
}

\section{(The utilization of the thermal lens technique for the analysis of thermal properties of transparent materials)}

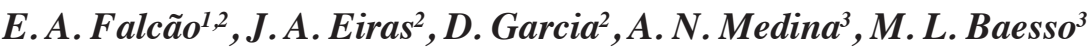 \\ ${ }^{I}$ Faculdade de Ciências Exatas e Tecnologia - FACET, Universidade Federal da Grande Dourados, Rod. \\ Dourados-Itahum, km 12, C.P. 533, Dourado, MS 79804-970 \\ ${ }^{2}$ Departamento de Física, Universidade Federal de S. Carlos, Rod. Washington Luiz, km 235, \\ S. Carlos, SP 13565-905 \\ ${ }^{3}$ Departamento de Física, Universidade Estadual de Maringá, Av. Colombo 5790, Maringá, PR 87020-900 \\ evaristofalcao@yahoo.com.br
}

\begin{abstract}
Resumo
A difusividade térmica é uma das propriedades físicas que está entre as mais importantes em um material. Sua determinação apresenta inúmeras dificuldades, o que requer o uso de técnicas precisas que forneçam valores confiáveis. Neste trabalho será apresentado à viabilidade de utilização de espectroscopia de lente térmica (ELT) na determinação da difusividade térmica (D) de materiais transparentes. Como exemplo discute-se os valores da difusividade térmica de materiais policristalinos, vítreos, poliméricos e cristais líquidos. Quando comparada com técnicas convencionais de medidas de difusividade térmica, a ELT apresenta a vantagem de ser rápida, medir amostras de tamanho reduzido, e permitir a obtenção de valores precisos em temperatura ambiente. Quando usada com outras técnicas permite a determinação de outros parâmetros térmicos e ópticos. Ou seja, ELT é uma poderosa ferramenta na determinação de parâmetros termo-ópticos de materiais transparentes.
\end{abstract}

Palavras-chave: difusividade térmica, espectroscopia de lente térmica, materiais transparentes.

\begin{abstract}
The thermal diffusivity is one of the physical properties that are among the most important in a material. Its determination presents several difficulties that require the use of accurate techniques that supply trustworthy values. In this work will be present the utilization of the thermal lens spectroscopy (TLS) as a technique to determine the thermal diffusivity (D) of transparent materials. As example are discussed the values of the thermal diffusivity of polycrystalline materials, glasses, polymer and liquid crystals. When compared with conventional techniques, the TLS presents the advantage to be rapid, to measure small sample size and to give accurate values at room temperature in the process of determination of the thermal diffusivity. When in concomitant use with other techniques it allows the determination of other thermo-optical parameters. In other words, the thermal lens spectroscopy is a powerful tool in the determination of thermo-optical parameters of transparent materials.
\end{abstract}

Keywords: thermal diffusivity, thermal lens spectroscopy, transparent ferroelectric ceramics.

\section{INTRODUÇÃO}

A medida da difusividade é uma das mais importantes em problemas que envolvem estados não estacionários de trocas de calor. Muitas são as técnicas experimentais utilizadas para obtenção de valores precisos deste parâmetro em materiais. Estando entre as técnicas mais utilizadas, para o caso de materiais cerâmicos, o método do fio quente e a técnica de pulso de energia $[1,2]$. O método do fio quente apesar de amplamente utilizado, apresenta limitações quanto ao tamanho da amostra e intervalo de tempo entre as medidas que chega a ser de até $10 \mathrm{~h}$ [1]. Já na técnica de pulso de enegia o intervalo de tempo entre as medidas e as dimensões das amostras utilizadas são menores que no método de fio quente. Porém, em tal técnica medidas a temperatura ambiente são muito difíceis de serem realizadas uma vez que a temperatura ambiente é sempre detectada pelo detector de infravermelho, o que por sua vez exerce influências sobre os resultados experimentais [2]. Por sua vez, há uma técnica experimental descoberta na década de 60 [3] que tem se mostrado altamente sensível na determinação de parâmetros termo-ópticos de materiais transparentes. Esta técnica, conhecida como espectrocopia de lente térmica (ELT), permite a obtenção de valores precisos da difusividade térmica sendo aplicada a materiais transparentes na região visível do espectro, até mesmo, materiais com baixos 
coeficientes de absorção, da ordem de $10^{-7} \mathrm{~cm}^{-1}$. A ELT é uma técnica não destrutiva e de caráter remoto, ou seja, não exige contato entre a amostra e o detector o que possibilita a realização de medidas em função da temperatura de modo a estudar as transições de fase que possam ocorrer. Esta técnica já foi aplicada com sucesso no estudo de materiais como cristal líquido [4], cerâmicas [5], monocristal de KDP [6], vidros [7], polímeros [8], óleo de soja [9], água [10], entre outros materiais. Apesar da ELT não medir o valor do calor específico e condutividade térmica, como no caso do método de fio quente e pulso de energia, o intervalo de tempo e tamanho de amostra são significativamente reduzidos. Outra vantagem de se utilizar a ELT para medir a difusividade térmica em materiais transparentes é que, valores precisos de difusividade térmica em temperatura ambiente podem ser obtidos.

Neste trabalho são apresentados e avaliados resultados experimentais de medidas de difusividade em materias policristalinos, cristalinos, vítreos, poliméricos, cristais líquidos e etc. Mostrando que a ELT é uma poderosa ferramenta na determinação de parâmetros térmicos. Será mostrado ainda que a ELT quando em uso concomitante com outras técnicas experimentais como calorimetria de relaxação térmica (CRT) e interferometria óptica (IO) permite a determinação completa dos parâmetros térmicos e ópticos tais como a condutividade térmica, coeficiente da temperatura da mudança do comprimento do caminho e o coeficiente da temperatura do índice de refração.

\section{EXPERIMENTAL}

\section{Lente térmica}

Em 1964 um grupo de pesquisadores, entre eles os brasileiros R. C. C. Leite e S. P. S. Porto, nos laboratórios da Bells Telefone observaram um efeito não linear na divergência do feixe ao introduzir amostras de corante dentro da cavidade de um laser de $\mathrm{HeNe}$ [3]. O efeito manifestava-se como um transiente da intensidade do laser e após um tempo, da ordem de segundos, o diâmetro de feixe era maior. Eles associaram este efeito a uma lente e a partir disto ficou conhecido como efeito de "Lente Térmica" (LT). Basicamente o efeito de LT tem como princípio a variação do índice de refração com a temperatura $[11,12]$. Quando um feixe laser com perfil de intensidade gaussiano passa através da amostra, a energia absorvida é convertida em calor que provoca uma mudança radial no índice de refração fazendo a amostra se comportar como uma lente. $\mathrm{O}$ aquecimento local faz com que o feixe sofra uma distorção ao passar pela amostra, que por sua vez produz uma mudança no comprimento do caminho óptico ds/dT podendo ser positiva (ds/dT) $>0$ ou negativa $(\mathrm{ds} / \mathrm{dT})<0$ dependendo das propriedades físicas da amostra. $\mathrm{O}$ termo ds/dT descreve a distorção termicamente induzida do feixe laser e carrega informação sobre o coeficiente térmico do índice de refração dn/dT. O conhecimento das propriedades térmicas e ópticas é essencial para avaliação da figura de mérito de materiais transparentes. Elas podem ser usadas para determinar as condições de trabalho como choque térmico, resistência ao stress térmico e biestabilidade óptica. Desde a descoberta do efeito de LT, muitos modelos foram desenvolvidos. Até o momento, o modelo teórico mais sensível para a ELT é o aberrante no modo descasado [13, 14]. Tal modelo leva em consideração a aberração esférica durante a formação da lente térmica (LT) e dá a diferença de fase do feixe de prova, induzida pela LT, que está relacionada com os parâmetros térmicos e ópticos da amostra tais como a difusividade térmica (D), a condutividade térmica $(\mathrm{k})$, absorção óptica $\left(\mathrm{A}_{\mathrm{e}}\right)$ e coeficiente da temperatura do comprimento do caminho óptico (ds/dT). No arranjo experimental de dois feixes no modo descasado Fig. 1, o valor da difusividade térmica é obtido pela equação A $[13,14]$.

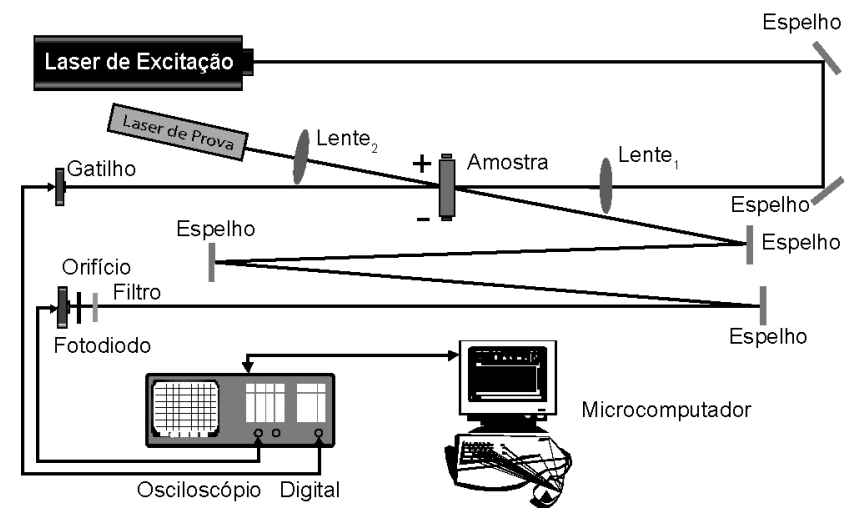

Figura 1: Configuração experimental para realização das medidas de lente térmica com duplo feixe no modo descasado.

[Figure 1: Experimental setup to the thermal lens spectroscopy with two beams in mismatched mode].

$$
D=\left(\frac{\omega_{e}^{2}}{4 t_{c}}\right)
$$

Em que $t_{c}$ é o tempo característico de formação da lente térmica e $\omega_{\mathrm{e}}$ é raio do laser de excitação em sua cintura. $\mathrm{O}$ valor de $\mathrm{t}_{\mathrm{c}}$ é obtido a partir do ajuste teórico dos dados experimentais pela seguinte expressão analítica (equação B)

$$
\begin{aligned}
& I_{p}(t)=I_{p}(0)\left\{1-\frac{\theta}{2} \tan ^{-1}\left[\frac{2 m V}{\left((1+2 m)^{2}+V^{2}\right)(t / 2 t)+1+2 m+V^{2}}\right]\right\} \\
& \theta=-\frac{P_{e} A_{e} l_{0}}{k \lambda_{p}}\left(\frac{d S}{d T}\right)_{L T} \\
& m=\left(\frac{\omega_{p}}{\omega_{e}}\right) ; V=\frac{Z_{1}}{Z_{c}} \operatorname{com~} Z_{c}=\left(\Pi w_{o p}{ }^{2}\right) / \lambda_{p}
\end{aligned}
$$


Nas equações acima $I_{p}(t)$ é a dependência temporal do laser de prova no detector, $\mathrm{I}_{\mathrm{p}}(0)$ é o valor inicial antes da formação da LT, $\theta$ é aproximadamente a diferença de fase termicamente induzida do feixe de prova após passar pela amostra, $\mathrm{P}_{\mathrm{e}}$ é a potência do feixe de excitação, $A_{e}$ é o coeficiente de absorção da amostra para o comprimento de onda do feixe de excitação, $1_{0}$ é a espessura da amostra, $\mathrm{k}$ é a condutividade térmica, $\lambda_{\mathrm{p}}$ é o comprimento de onda do feixe de prova, $(\mathrm{ds} / \mathrm{dT})_{\mathrm{LT}}$ é a mudança do coeficiente da temperatura do comprimento do caminho óptico, [13, 14]. Nas medidas de resolução temporal, $\theta$ é obtido a partir do ajuste dos dados experimentais pela equação $\mathrm{B}, Z_{c}$ é a distância confocal do feixe de prova, $Z_{1}$ é a distância entre a cintura do feixe de prova e a amostra, $Z_{2}$ é a distância entre a amostra e o fotodiodo, $\omega_{\mathrm{p}}$ e $\omega_{\mathrm{e}}$ são os raios dos lasers de prova e excitação na amostra, respectivamente. Devido ao caráter remoto as medidas podem ser realizadas em função da temperatura ou de outros sinais de excitação. No arranjo experimental de duplo feixe no modo descasado são utilizados dois lasers com diâmetros diferentes na amostra um para excitar e outro para provar o efeito, as medidas são realizadas de modo a ter um curto tempo de exposição da amostra no modo de resolução temporal para obtenção da difusividade térmica [16]. Em um arranjo experimental típico a amostra é colocada na cintura do feixe de excitação, onde a potência é máxima, e na posição confocal do feixe de prova $[11,16]$. Na ordem de obter o sinal de lente térmica, um obturador mecânico controla a exposição da amostra ao feixe de excitação. Um colimador é colocado na frente do fotodiodo para selecionar somente a parte central do feixe de prova. Um segundo fotodiodo é usado para dar início à aquisição dos dados experimentais por um osciloscópio digital, os dados obtidos pelo osciloscópio são armazenados em um micro computador, por um programa de aquisição para análise posterior. A potência do feixe de excitação varia de acordo com a absorção da amostra.

\section{Materiais}

Os resultados experimentais que serão apresentados referem-se à água cujo dado experimental foi retirado da Ref. [19], às cerâmicas policristalinas transparentes, dados retirados das Ref. [15, 18], aos materiais vítreos, dados retirados da Ref. [7], ao polímero, dado retirado da Ref. [20] e ao cristal líquido dado retirado da Ref. [4]. Maiores detalhes quanto ao processamento, das amostras podem ser vistos nas referências acima.

\section{RESULTADOS E DISCUSSÃO}

Em alguns dos trabalhos citados anteriormente que compõe esta revisão, as medidas foram realizada em função da temperatura de modo a se ter o comportamento das propriedades termo-ópticas nas mesmas condições de trabalho a que eles estariam sujeitos, a fim de se detectar possíveis transições de fase que pudessem ocorrer durante o

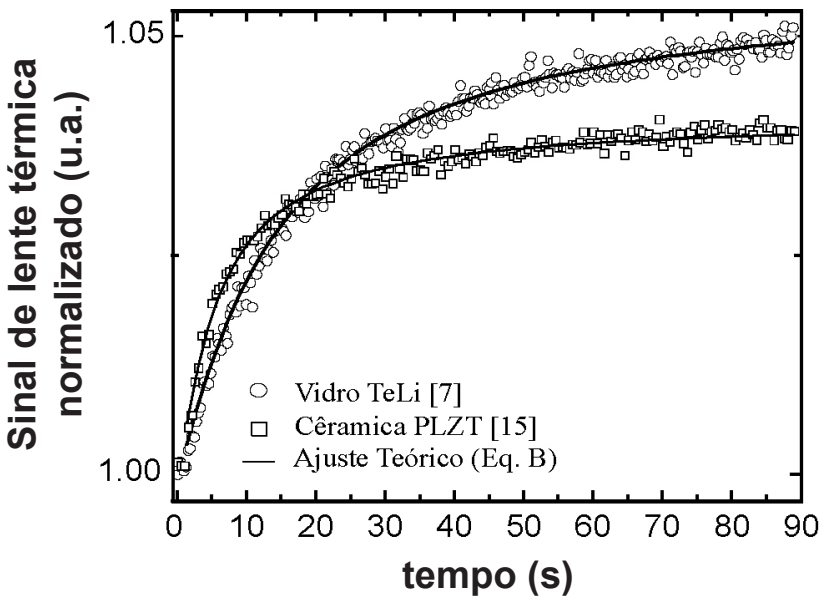

Figura 2: Curva transiente típica de LT no modo descasado para as amostras de cerâmica transparente PLZT ( $\square$ ) (15) e vidro telureto TeLi ( $\square$ ) (7) em temperatura ambiente.

[Figure 2: Typical transient curve of TL in the mismatched mode for the transparent ferroelectric ceramic sample PLZT (ㅁ) (15) and tellurite glass TeLi ( $\square$ (7) at room temperature.]

intervalo de medida. Porém, por simplicidade optamos por apresentar apenas os resultados em temperatura ambiente, uma vez que os trabalhos originais podem ser consultados para maiores detalhes. A Fig. 2 mostra uma curva transiente típica de LT para as amostras de cerâmica policristalina (PLZT 10/65/35) e vidro telureto (TeLi), em que a partir do ajuste teórico dado pela equação $\mathrm{B}$, foram obtidos os seguintes valores $\theta=(-32,8 \pm 0.2) \times 10^{-3} \mathrm{rad}, \mathrm{t}_{\mathrm{c}}=(0,82 \pm 0,01) \times 10^{-3} \mathrm{~ms}$ para a cerâmica [15] e $\theta=-(50,8 \pm 0,2) \times 10^{-3} \mathrm{rad}, \mathrm{t}_{\mathrm{c}}=(2,12 \pm 0,01)$ $\mathrm{x} 10^{-3} \mathrm{~ms}$ para o vidro telureto [21]. A partir destes valores e através do uso da equação A os autores obtiveram os seguintes resultados para difusividade térmica da cerâmica $\mathrm{D}=(7,0 \pm 0,1) \times 10^{-3} \mathrm{~cm}^{2} / \mathrm{s} \mathrm{e} \mathrm{D}=(2,7 \pm 0,1) \times 10^{-3} \mathrm{~cm}^{2} / \mathrm{s}$. No trabalho da Ref. [15] foi observado que o valor de difusividade térmica para cerâmica policristalina (PLZT 10/65/35) era $40 \%$ maior que o obtido na Ref. [18] (PLZT 11/65/35), os autores atribuíram essa diferença a composição e processamento do material. Para efeito de comparação esses resultados estão na Tabela I. Para o caso do trabalho da Ref. [21] os autores valores de difusividade térmica muito próximos para vidros calcogeneto (GaLaS) similares [21]. No trabalho da amostra de policarbonato (material polimérico) foi encontrado um valor de difusividade térmica $\mathrm{D}=(0,99 \pm 0,03) \times 10^{-3} \mathrm{~cm}^{2} / \mathrm{s}$ (Tabela II) [20]. Na Ref. [20] os autores optaram por dar ênfase à região de temperatura entre 120 e $170{ }^{\circ} \mathrm{C}$ de modo a quantificar a temperatura de transição vítrea $(\mathrm{Tg})$ do material e comparam os resultados obtidos com medidas de calorimetria de varredura diferencial (DSC). No trabalho do cristal líquido [4] os autores mostraram a sensibilidade da técnica para detectar transições de fase muito estreita, da ordem de $1{ }^{\circ} \mathrm{C}$ a meia altura. Após o alinhamento da amostra, com um campo magnético externo aplicado realizaram as medidas na direção ordinária e extraordinária respectivamente [4]. Os valores obtidos em temperatura ambiente foram $\mathrm{D}_{\square} \approx 1,7 \times 10^{-3} \mathrm{~cm}^{2} / \mathrm{s}$ e $\mathrm{D}_{\square} \approx 1,4 \times 10^{-3} \mathrm{~cm}^{2} / \mathrm{s}$, conforme 
Tabela I - Valores da difusividade térmica da cerâmica PLZT e vidro telureto.

[Table I - Values of the thermal diffusivity of the PLZT ceramic and tellurite glass.]

\begin{tabular}{lc}
\hline Amostras & $\mathrm{D} \mathrm{x} 10^{-3} \mathrm{~cm}^{2} / \mathrm{s}$ \\
\hline $\begin{array}{l}\text { PLZT 10/65/35 (cerâmica } \\
\text { policristalina) (6) }\end{array}$ & $7,0 \pm 0,1$ \\
PLZT 11/65/35 (cerâmica & \\
policristalina) (18) & 5 \\
TeLi (vidro) (7) & $2,12 \pm 0,01$ \\
\hline
\end{tabular}

Tabela II - Valores da difusividade térmica do policarbonato, cristal líquido e água.

[Table II - Values of the thermal diffusivity of the polycarbonate, liquid crystal and water.]

\begin{tabular}{|c|c|}
\hline Amostras & $\mathrm{D} \times 10^{-3} \mathrm{~cm}^{2} / \mathrm{s}$ \\
\hline Policarbonato (polímero) (20) & $0,99 \pm 0,03$ \\
\hline $\begin{array}{ll}\text { Cristal Líquido (4) } & \mathrm{D}_{\|} \\
& \mathrm{D}_{\perp}\end{array}$ & $\begin{array}{c}2 \\
1,4\end{array}$ \\
\hline Água (19) & $1,33 \pm 0,08$ \\
\hline
\end{tabular}

Tabela II. Apesar do modelo não ter sido desenvolvido para materiais anisotrópicos é sensível o suficiente para realizar medidas em diferentes direções do material. No trabalho da água os autores [19] obtiveram um valor para difusividade térmica $\mathrm{D}=(1,33 \pm 0,08) \times 10^{-3} \mathrm{~cm}^{2} / \mathrm{s}$ valor que apresenta boa concordância com a literatura [22] que é $\mathrm{D}=1,4 \times 10^{-3} \mathrm{~cm}^{2} / \mathrm{s}$. Este resultado é de fundamental importância, pois mostra a reprodutibilidade e a confiabilidade dos resultados de difusividade térmica obtidos via ELT. Os autores ainda reportam anomalias observadas no transiente de lente térmica e atribuíram este comportamento a reação fotoquímica.

Outra vantagem de se utilizar a ELT para obtenção da difusividade térmica é que, em combinação com a CRT e IO outros parâmetros podem ser obtidos dando assim uma caracterização completa das propriedades térmicas e ópticas dos materiais. Optamos por apresentar apenas os resultados obtidos para cerâmica policristalina PLZT 10/65/35. Porém, este estudo se aplica a outros materiais transparentes. Maiores detalhes da combinação das três técnicas podem ser vistos nas Refs. $[7,15]$. Para a cerâmica o valor do calor específico obtido pela CRT foi de $c_{p}=(0,36 \pm 0,01) \mathrm{J} / \mathrm{gK}$, que combinado com o valor da difusividade e através da relação $\mathrm{k}=\mathrm{D} \rho \mathrm{c}_{\mathrm{p}}$ foi obtido um valor de $\mathrm{k}=(18 \pm 3) \times 10^{-3} \mathrm{~W} / \mathrm{cmK}$. Das medidas de interferometria óptica o valor encontrado para o coeficiente da temperatura da mudança do comprimento do caminho óptico foi $(\mathrm{ds} / \mathrm{dT})_{\mathrm{IO}}=(8,5 \pm 0,3) \times 10^{-5} \mathrm{~K}^{-1}$ (via interferometria óptica) que combinado com o valor do coeficiente de expansão linear $(\alpha)$ e através da relação $(\mathrm{ds} / \mathrm{dT})_{\mathrm{IO}}=\mathrm{dn} / \mathrm{dT}+\mathrm{n} \alpha$ os autores estimaram o valor do coeficiente da temperatura do índice de refração dn/dT $=(7,2 \pm 0,3) \times 10^{-5} \mathrm{~K}^{-1}[15]$.

\section{CONCLUSÕES}

Foi feita uma descrição simplificada do modelo de lente térmica no modo descasado. Foi mostrado, a partir de dados experimentais retirados da literatura, que a lente térmica permite a obtenção de valores precisos de difusividade térmica em materiais transparentes. Foi mostrado ainda que a espectroscopia de lente térmica permite a obtenção de valores precisos em temperatura ambiente. Foi mostrado também que lente térmica combinada com outras técnicas experimentais permite a obtenção de outros parâmetros termo-ópticos. Finalmente foi mostrado que a lente térmica é uma poderosa ferramenta na análise das propriedades termo-ópticas de materiais transparentes.

\section{AGRADECIMENTOS}

Às agências FAPESP, CNPq, CAPES e Fundação Araucária pelo suporte financeiro.

\section{REFERÊNCIAS}

[1] W. N. Santos, Polímeros: Ciência e Tecnologia 15 (2005) 289.

[2] W. N. Santos, Cerâmica 51 (2005) 205.

[3] J. P. Gordon, R. C. C. Leite, R. S. Moore, S. P. S. Porto, J. R. Whinnery, Bull. Am. Phys. Soc. 9 (1964) 501.

[4] J. R. D. Pereira, A. J. Palangana, A. C. Bento, M. L. Baesso, A. M. Mansanares, E. C. Silva, Rev. Sci. Instr.74 (2003) 822.

[5] T. Chen, S. J. Sheih, J. F. Scott, Phys. Rev. B 43 (1991) 615.

[6] A. E. Falcão, A. Steimacher, A. N. Medina, J. R. D. Pereira, A. C. Bento, C. M. R. Remedios, J. M. Sasaki, J. Physique IV 125 (2005) 391.

[7] S. M. Lima, W. F. Falco, E. S. Bannwart, L. H. C. Andrade, R. C. Oliveira, J. C. S. Moraes, K. Yukimitu, E. B. Araújo, E. A. Falcão, A. Steimacher, N. G. C. Astrath, A. C. Bento, A. N. Medina, M. L. Baesso, J. Non Crist. Solids 352 (2006) 3603.

[8] J. H. Rohling, J. R. D. Pereira, A. N. Medina, A. C. Bento, M. L. Baesso, J. A. Sampaio, A. M. Lima, T. Catunda, L. C. Miranda, Rev. Sci. Instr. 74 (2003) 291.

[9] T. A. S. Albuquerque, P. R. B. Pedreira, A. N. Medina, J. R. D. Pereira, A. C. Bento, M. L. Baesso, Rev. Sci. Instr. 74 (2003) 694.

[10] P. R. B. Pedreira, L. R. Hirsch, J. R. D. Pereira, A. N. Medina, A. C. Bento, M. L. Baesso, M. C. E. Rollemberg, M. Franko, Chem. Phys. Lett. 396 (2004) 221.

[11] M. L. Baesso, J. Shen, R. D. Snook , J. Appl. Phys. 75, 8 (1994) 3732.

[12] J. P. Gordon, R. C. C. Leite, R. S. More, S. P. S. Porto, J. Appl. Phys. 36 (1965) 3.

[13] S. Jun, R. D. Lowe, R. D. Snook, Chem. Phys. 165, 2-3 (1992) 385 . 